\title{
Deep Learning Based QRS Multilead Delineator in
}

\section{Electrocardiogram Signals}

\author{
Julià Camps ${ }^{1}$, Blanca Rodríguez ${ }^{1}$, Ana Mincholé ${ }^{1}$ \\ ${ }^{1}$ Department of Computer Science, University of Oxford, Oxford, UK
}

\begin{abstract}
The surface electrocardiogram (ECG) is the most widely adopted test to diagnose cardiac diseases. Extracting critical biomarkers from these signals, such as the QRS width, requires delineating the fundamental waves in them. However, even though ECG signals significantly change depending on the recording methodology and cardiac condition, the available QRS delineators are hard to adapt to non-considered cases. We present a deep learning-based multilead ECG delineation method which can successfully delineate QRS complexes. Our approach reached root-mean-square errors (RMSE) of $12.1 \pm 0.5$ and $18.5 \pm 1.1 \mathrm{~ms}$ for $Q R S$ onset and offset, respectively, when evaluated on the $Q T$ database; thus, demonstrating to be comparable to the state-of-the-art. Moreover, these results are similar to the RMSE calculated from differences between the two cardiologists that annotated this database, namely, 14.7 ms for the QRS onset and 17.2 ms for the offset.
\end{abstract}

\section{Introduction}

According to the World Health Organization [1], cardiac diseases killed more than 9 million people in 2016 and are considered the primary cause of death worldwide. The surface electrocardiogram (ECG) is the most popular non-invasive test employed in clinical practice to diagnose and monitor cardiac diseases. The ECG provides signal-data of the heart's electrical activity. Analysing these signals requires delineating the main waves composing the beats. More precisely, the ECG delineation process involves identifying the exact positions of the onset, offset and peak of the following waveforms: P-wave, QRS-complex and T-wave to calculate representative biomarkers.

Manually delineating ECG recordings is repetitive and time-consuming. Therefore, some automatic delineation methods have been proposed in the literature [2]-[9]. While there exist both, rule-based [2]-[6] and machine learning-based QRS delineators [7]-[9], the state-of-theart are the former [3], [6]. However, these methods lack adaptability to significant differences in the target scenario since the rules composing them have been carefully designed by experts to match a specific domain, for example, a database comprising ECG signals recorded from athletes while exercising. Indeed, adapting these solutions to non-planned scenarios requires expertknowledge about the task and the system's design, which has limited the acceptance of the existing QRS delineation approaches. On the other hand, deep learning models have the potential to automatically adapt to the training-data's domain. Thus, if an appropriate dataset is available deep learning methods can be automatically adapted to any domain.

Deep learning is a subclass of machine learning techniques which are capable of automatically extracting the feature representation from the data without prior expert knowledge [10]. Deep learning techniques have shown to outperform the state-of-the-art in several complex tasks, such as text translation [11]. In fact, they have even outperformed humans in image classification [12], lip reading [13] and playing games [14]. Deep learning techniques require large datasets to learn appropriate representations about the target task. Consequently, these methods are typically combined with data augmentation strategies. Data augmentation strategies generate artificial variants of the samples in the data to improve the exploitation efficiency. 


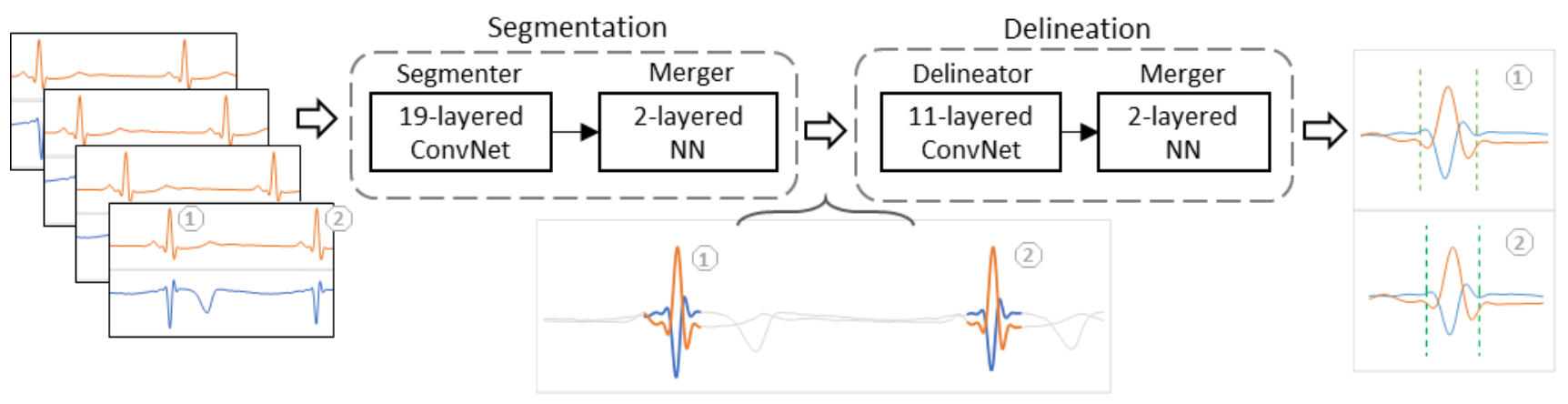

Figure 1. Delineation system diagram formed by a segmentation and a delineation modules, each composed of a ConvNet and a NN. The first module identifies the QRSs, and the second one locates the QRS's onsets and offsets, respectively.

We present a novel two-stepped deep learning-based multilead QRS delineation system. This system first segments the QRS waves from the ECG recording and then delineates them. More precisely, our approach is formed by successive segmentation and delineation modules, each of which is composed of a onedimensional (1D) convolutional neural network (ConvNet) and a fully-connected neural network (NN). To validate our approach we used nested cross-validation (CV) on the QT database (QTDB) [15]. Previous studies have addressed ECG segmentation and detection [16]. Nevertheless, to our knowledge, this is the first work to propose a deep learning-based QRS delineator.

\section{Methods}

We propose a delineator system formed by two modules, each of which is composed of two deep learning models (see Figure 1). Figure 1 illustrates these two modules and how the system works. Firstly, the segmentation module, formed by the segmenter and segmentation merger, locates the QRS waves in the ECG signal. Then, the data are windowed again using only areas containing a QRS complex. Finally, the delineation module, which is formed by the delineator and delineation merger, defines the QRS onset and offset marks.

\subsection{Architecture}

The architectures proposed are hereafter outlined. The segmenter (see Figure 2) is a 19-layered 1D ConvNet composed of 16 convolutional and three fully-connected layers. Each convolutional layer is formed by 16 kernels of length 3 . These layers implement batch normalisation and use rectified linear unit (ReLU) activation functions. Next, the two hidden fully-connected layers have 128 and 256 neurons, respectively. Furthermore, both layers implement batch normalisation, dropout at a rate of 0.5 and ReLU as the activation functions. Finally, the output layer was another fully-connected layer with as many sigmoid units as the size of the input window to enable the model to perform classification from one-hot encoded labels.

The second model of the segmentation module is the segmentation merger. This model is a 2-layered NN composed of two fully-connected layers, with 512 and one neurons in the first and second layer, respectively. The first layer implements batch normalisation, dropout at rate 0.5 and ReLU as the activation functions. There is a sigmoid output layer.

Analogously to the segmentation module, the delineator and the segmentation merger are also a 1D ConvNet and an NN, respectively. Except for having 8 convolutional layers with kernels of size 5, the delineator is composed of the same elements than the segmenter. On the other hand, the delineation merger is identical to the segmentation one.

The number of layers and kernel sizes were chosen to enable our models to learn patterns with length up to 33 instances (i.e. $132 \mathrm{~ms}$ ), which accounts for most QRS morphologies. 


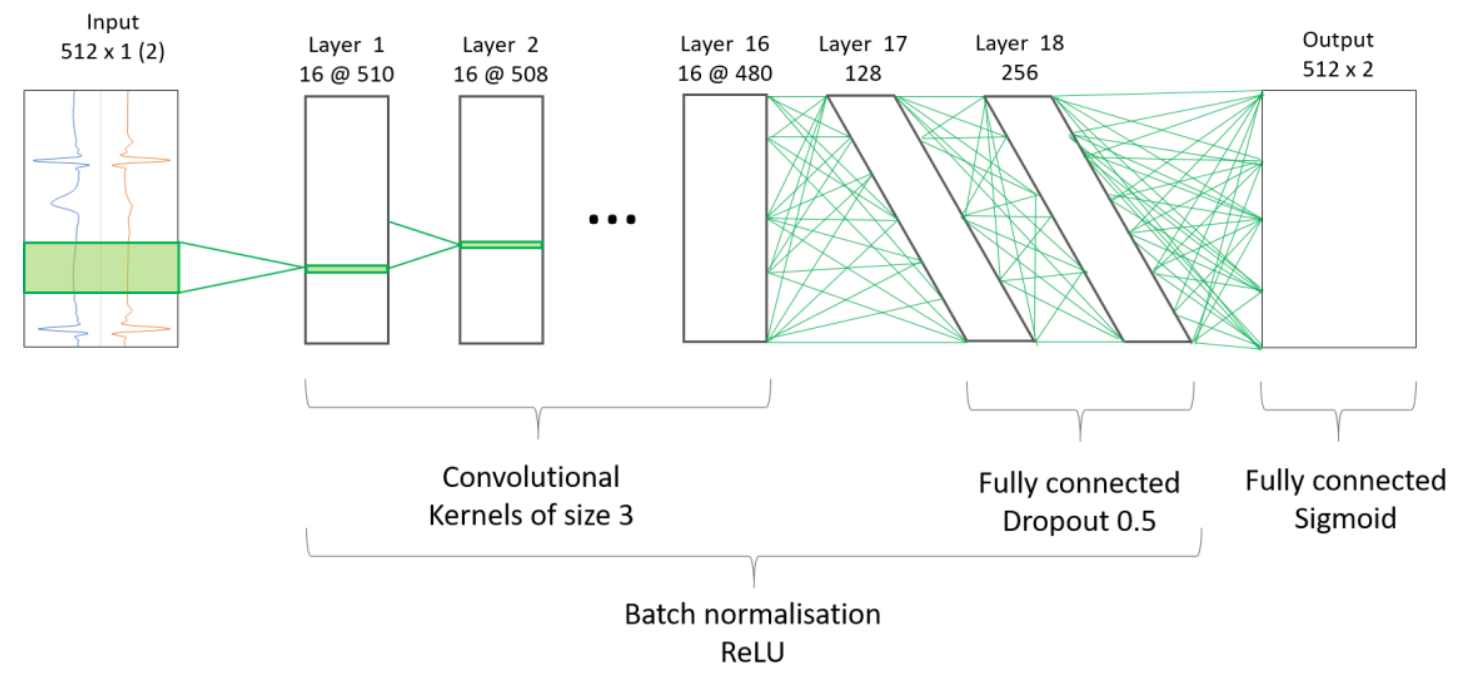

Figure 2. Segmenter model diagram which is a 19-layered 1D ConvNet composed of 16 convolutional and 3 fully-connected layers.

\subsection{Data}

The proposed method was validated using the QTDB. This database includes 105 two-lead ECG recordings at $250 \mathrm{~Hz}$ sampling rate from 7 databases (e.g. the MIT-BIH Arrhythmia database and the European ST-T database).

These signals were windowed at 2.048 s (i.e. 512 instances); thus, according to RR-interval known ranges, there should be at least one QRS complex in each sample. Then each window was normalised by subtracting the mean of its values and dividing by their standard deviation.

The augmentation strategies implemented to train our models implied using 99\%-overlapping when windowing the samples, and adding constants and scaling the values of the ECG signals.

\subsection{Methodology}

Firstly, the segmenter trains using the augmented and windowed data. Secondly, it predicts segmentation labels on each recording using a sliding window with jumps of $1 \%$ instances. Due to the sliding strategy, this process generates a matrix of predictions. Next, the segmentation merger takes this matrix and trains on how to combine these predictions. Once trained, this merger computes a single segmentation prediction for every instance in the recording. Then, the system windows the segments of the signal with a QRS to $256 \mathrm{~ms}$ to give some margin to the identified zones. Later, the delineator trains on the task of finding the onset and offset marks using these shorter windows generated by the segmentation module, each of which contains only one QRS complex. Moreover, these samples are augmented again to train the delineator. Once trained, analogous to the segmentation module, the delineator will produce a matrix of predictions using a sliding window strategy. Then, the delineator merger uses this matrix to compute the QRS onset and offset positions.

\subsection{Training and evaluation}

Some hyperparameters, namely the number of convolutional layers, the kernel sizes and the window lengths, were chosen according to domain knowledge about the of ECG signals and its waves. Furthermore, we used Adam [17] and binary cross-entropy since this task is a one-hot-encoding binary classification problem due to its resemblance to segmentation.

We chose the remaining hyperparameters and validated the system using nested CV with leave-onedatabase-out and grid search. More precisely, each fold included the recordings from one of the databases included in the QTDB. Then testing performances were computed each time for one fold using leave-onedatabase-out cross-validation with the others to choose the hyperparameters and learn the models' weights. Consequently, the performances reported are an approximation of how our approach would generalise to other databases. Moreover, the training and assessment were repeated 10 times to assess the reproducibility.

The performance measurements employed in this study were the root-mean-square error (RMSE), the mean error (ME), the error's sample standard deviation (STE) and the QRS miss rate. The equations for these metrics are $R M S E=\sqrt{\sum_{i=1}^{n}\left(\hat{y}_{i}-y_{i}\right)^{2} / n}, \quad M E=\sum_{i=1}^{n}\left(\hat{y}_{i}-y_{i}\right) / n$, and $S T=\sqrt{\sum_{i=1}^{n}\left(\left(\hat{y}_{i}-y_{i}\right)-M E\right)^{2} / n-1}$. Where $n$ is the number of samples and, $\hat{y}_{i}$ and $y_{i}$ are the predicted and the real labels of the $i$ th sample, respectively. The QRS miss rate is the number of missed plus the number of wrongly found QRS waves during the segmentation 
phase, divided by the total number of QRS complexes.

\section{Results and discussion}

The RMSE of our approach was $12.1 \pm 0.5 \mathrm{~ms}$ and $18.5 \pm 1.1 \mathrm{~ms}$ for the QRS onset and offset, respectively. The ME $\pm E S T D$ achieved was $-2.6 \pm 10.8 \mathrm{~ms}$ and $4.4 \pm 15.2$ $\mathrm{ms}$, for the onset and offset respectively. Whereas, [3] reported ME $\pm E S T D$ of $4.6 \pm 7.7 \mathrm{~ms}$ and $0.8 \pm 8.7 \mathrm{~ms}$ for the onset and offset, respectively; and [6] reported 7.0 \pm 4.3 $\mathrm{ms}$ and $-5.0 \pm 9.9 \mathrm{~ms}$ for the onset and offset, respectively.

The reference approaches are single-lead delineators [3], [6]. The performances of these methods in the QTDB were calculated from predicting for each lead independently and, later, fusing both outcomes by choosing the predicted value with less error for each timepoint. On the other hand, our approach is multilead; thus, it overcomes the need for selecting which predictions to use. Nevertheless, our performances are similar to the ones reported by the reference studies, which suggests that our approach is comparable to the state-of-the-art. Moreover, the RMSE calculated from the differences between the marks given by the two annotators in this database is $14.7 \mathrm{~ms}$ for the QRS onset and $17.2 \mathrm{~ms}$ for the offset, which helps to justify the magnitude of our errors.

\section{Conclusions}

We propose a deep learning-based multilead delineation system for QRS complexes in ECG signals. The implemented data augmentation strategies enabled our system to successfully learn from a scarce and highly diverse set of multilead ECG recordings how to delineate QRS complexes. Moreover, the method achieved comparable performances than the state-of-the-art.

\section{Acknowledgements}

The Engineering and Physical Sciences Research Council supports JC, and a Wellcome Trust Senior Research Fellowship in Basic Biomedical Sciences supports AM and BR.

\section{References}

[1] World Health Organization, "The top 10 causes of death," $2018 . \quad$ [Online]. Available: http://www.who.int/news-room/fact-sheets/detail/thetop-10-causes-of-death. [Accessed: 11-Aug-2018].

[2] P. Laguna, R. Jané, and P. Caminal, "Automatic detection of wave boundaries in multilead ECG signals: validation with the CSE database," Comput. Biomed. Res., vol. 27, no. 1, pp. 45-60, Feb. 1994.

[3] J. P. Martínez, R. Almeida, S. Olmos, A. P. Rocha, and P. Laguna, "A wavelet-based ECG delineator evaluation on standard databases," IEEE Trans.
Biomed. Eng., vol. 51, no. 4, pp. 570-581, 2004.

[4] R. Almeida, J. P. Martínez, a P. Rocha, and P. Laguna, "P wave delineation using spatially projected leads from wavelet transform loops," Comput. Cardiol. 2010, vol. XX, pp. 1003-1006, 2010.

[5] A. Martínez, R. Alcaraz, and J. J. Rieta, "A new method for automatic delineation of ECG fiducial points based on the Phasor Transform," 2010 Annu. Int. Conf. IEEE Eng. Med. Biol. Soc. EMBC'10, pp. 45864589, 2010.

[6] J. M. Bote, J. Recas, F. Rincon, D. Atienza, and R. Hermida, "A modular low-complexity ECG delineation algorithm for real-time embedded systems," IEEE $J$. Biomed. Heal. Informatics, vol. 22, no. 2, pp. 429-441, Mar. 2018.

[7] N. P. Hughes, L. Tarassenko, and S. J. Roberts, "Markov models for automated ECG interval analysis," Adv. Neural Inf. Process. Syst., vol. 16, pp. 611-618, 2004.

[8] S. Graja and J. M. Boucher, "Hidden Markov tree model applied to ECG delineation," IEEE Trans. Instrum. Meas., vol. 54, no. 6, pp. 2163-2168, 2005.

[9] G. De Lannoy, B. Frenay, M. Verleysen, and J. Delbeke, "Supervised ECG delineation using the wavelet transform and hidden markov models," IFMBE Proc., vol. 22, pp. 22-25, 2008.

[10] Y. Lecun, Y. Bengio, and G. Hinton, "Deep learning," Nature, vol. 521, no. 7553. pp. 436-444, 2015.

[11] N. Kalchbrenner, L. Espeholt, K. Simonyan, A. van den Oord, A. Graves, and K. Kavukcuoglu, "Neural machine translation in linear time," Arxiv, pp. 1-11, 2016.

[12] A. Krizhevsky, I. Sutskever, and G. E. Hinton, "ImageNet classification with deep convolutional neural networks," Adv. Neural Inf. Process. Syst., pp. $1-9,2012$.

[13] Y. M. Assael, B. Shillingford, S. Whiteson, and N. De Freitas, "LipNet: sentence-level lipreading," arXiv Prepr., pp. 1-12, 2017.

[14] D. Silver et al., "Mastering the game of Go without human knowledge," Nature, vol. 550, no. 7676, pp. 354-359, 2017.

[15] P. Laguna, R. G. Mark, A. Goldberg, and G. B. Moody, "A database for evaluation of algorithms for measurement of QT and other waveform intervals in the ECG," Comput. Cardiol. 1997, vol. 24, pp. 673676, 1997.

[16] Y. Xiang, Z. Lin, and J. Meng, “Automatic QRS complex detection using two-level convolutional neural network," Biomed. Eng. Online, vol. 17, no. 1, p. 13, Dec. 2018.

[17] D. P. Kingma and J. Ba, "Adam: A Method for Stochastic Optimization,” Arxiv, vol. abs/1412.6, 2014.

Address for correspondence.

Name. Julià Camps

Full postal address. Department of Computer Science, Parks Road, Oxford, OX1 3QD

E-mail address. julia.camps@cs.ox.ac.uk 